\title{
A case of double common bile duct visually detected only by cholangioscope
}

Double common bile duct is a rare congenital anomaly [1 -3$]$. We report a case of a 53-year-old Japanese man who was incidentally diagnosed with a double common bile duct by cholangioscope examination. He developed mild acute cholecystitis and received antibiotics. Drip-infusion cholangiography-computed tomography demonstrated a filling defect in the common bile duct (CBD) ( Fig. 1 a). Endoscopic retrograde cholangiography (ERC) showed a normal bile duct image ( $\vee$ Fig. $\mathbf{1 b}$ ), and the patient was diagnosed with spontaneous passage of choledocholithiasis. However, bile cytology detected atypical cells and this patient was referred to our facility. Contrast-enhanced endoscopic ultrasound showed no contrast echoic lesions in the cystic duct and gallbladder, and sludge was suspected, but the CBD was normal ( Fig.1c-e). We performed cholangioscopy (SpyGlass-DSII; Boston Scientific, Marlborough, Massachusetts, USA) to rule out minimal biliary cancer because previous cytology suggested possible malignancy. The cholangioscopy incidentally showed a common bile duct divided into two lumens ( $\vee$ Video 1 ). We inserted a guidewire from the contralateral lumen and visually recognized the crossed guidewire from the alternative lumen, which was evidence for a double common bile duct ( $\vee$ Fig. 2 a - c). The cystic duct branched from one lumen of the double CBD. The cholangioscopy did not show any tumor, and biopsy specimens and bile cytology were negative for malignancy.

This case is a type I double common bile duct according to Saito's classification [4]. There had previously been only one report of a double common bile duct visualized on cholangioscopy by Kawakubo [5], but this is the first report of a double common bile duct without ectopic drainage of the bile duct. Surprisingly, no

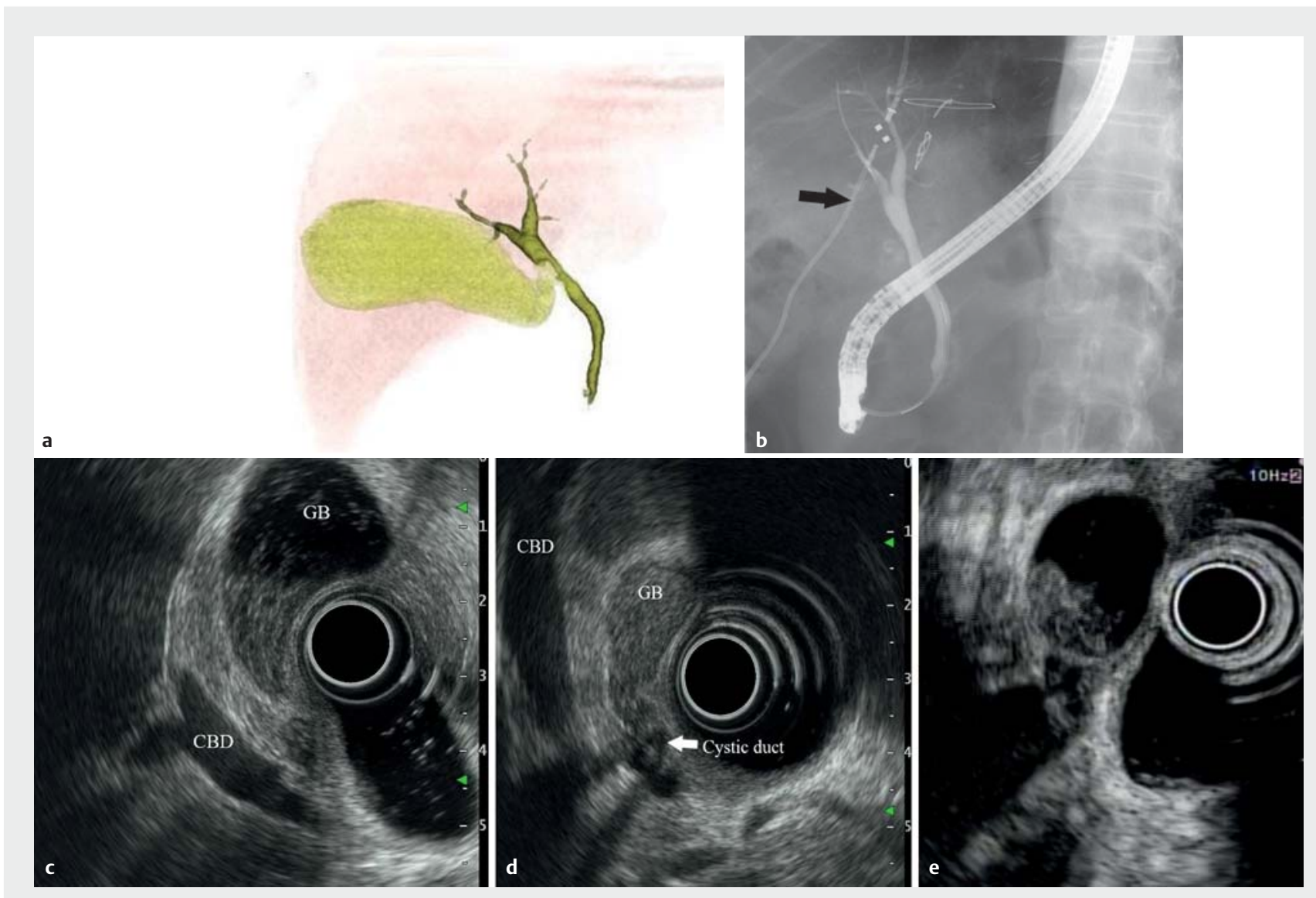

- Fig. 1 The imaging findings detected no double common bile duct. a Drip infusion cholangiography-computed tomography. b Endoscopic retrograde cholangiography. Black arrow shows a ventriculoperitoneal shunt for hydrocephalus. c, $\mathbf{d}$ Endoscopic ultrasound. e Contrast-enhanced endoscopic ultrasound. 

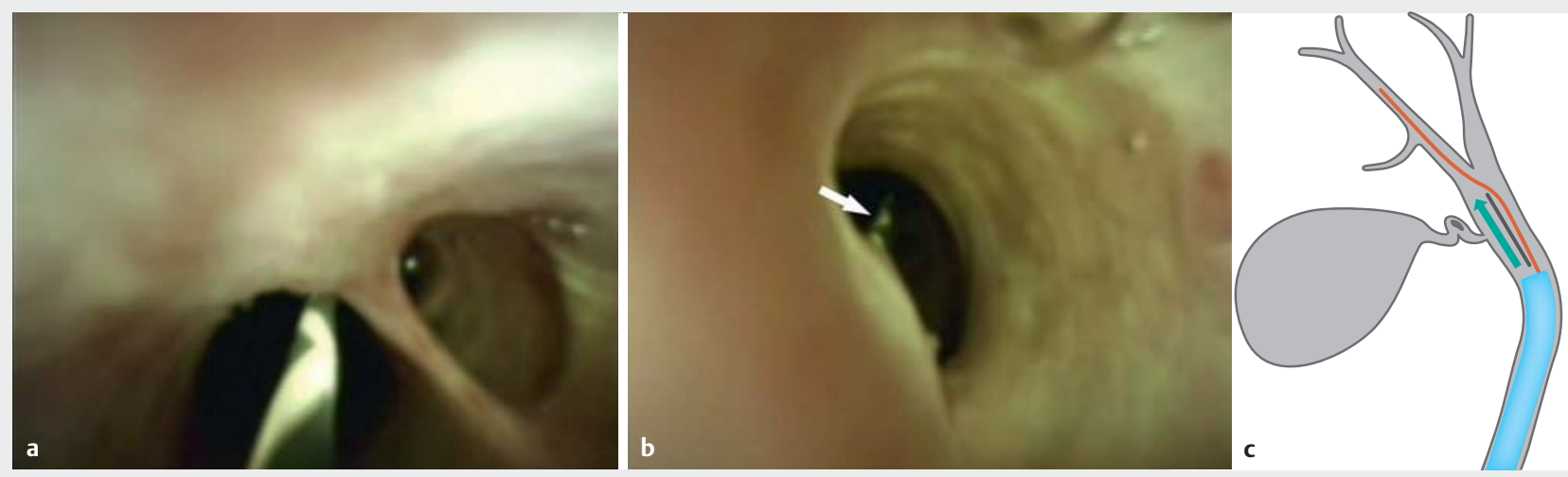

- Fig. 2 The double common bile duct visually detected by the cholangioscope. a, b View of the confirmation using a guidewire. White arrow shows the crossed guidewire. c Schema of the double common bile duct and confirmation using a guidewire. Black line shows the septum of the double common bile duct. Red line shows the guidewire. Arrow shows the view of the cholangioscope in $\mathbf{r i g} . \mathbf{2} \mathbf{b}$.

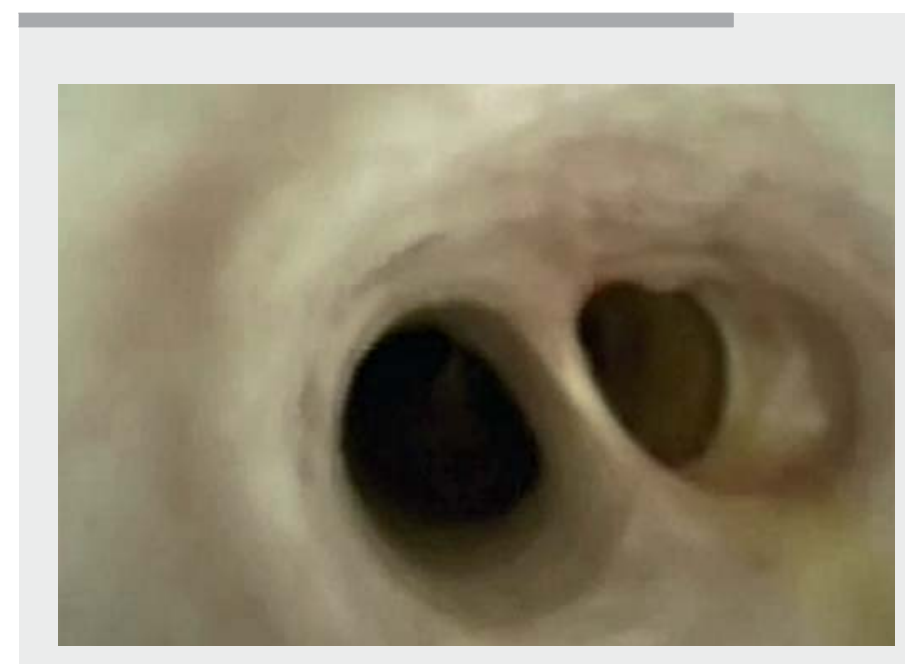

Video 1 A case of double common bile duct visually detected only by cholangioscope.

modality other than the cholangioscope could detect the double common bile duct. This finding indicates that type I cases might be more latent and prevalent. We hope that a greater number of cases will elucidate the epidemiology of the double common bile duct in future.

Endoscopy_UCTN_Code_TTT_1AR_2AB

\section{Competing interests}

The authors declare that they have no conflict of interest.
The authors

Shin Miura , Shin Hamada, Kiyoshi Kume, Kazuhiro Kikuta, Tetsuya Takikawa @ Naoki Yoshida, Atsushi Masamune

Division of Gastroenterology, Tohoku University

Graduate School of Medicine, Sendai, Japan

Corresponding author

\section{Shin Miura, MD}

Division of Gastroenterology, Tohoku University Graduate School of Medicine,

1-1 Seiryo-machi, Aoba-ku, Sendai, Miyagi, Japan

Fax: +81-22-717-7177

miurashin@med.tohoku.ac.jp

\section{References}

[1] Fan X, He L, Khadaroo PA et al. Duplication of the extrahepatic bile duct: a case report and review of the literatures. Medicine (Baltimore) 2018; 97: e9953

[2] Kolli S, Etienne D, Reddy M el al. A review of double common bile duct and its sequelae. Gastroenterology Res 2018; 11: 1-4

[3] Yamashita K, Oka Y, Urakami A et al. Double common bile duct: a case report and a review of the Japanese literature. Surgery 2002; 131: 676-681

[4] Saito N, Nakano A, Arase M et al. [A case of duplication of the common bile duct with anomaly of the intrahepatic bile duct]. Nihon Geka Gakkai Zasshi 1988; 89: 12961301

[5] Kawakubo K, Isayama H, Sasahira N et al. Double common bile duct visualized by SpyGlass cholangioscopy. Endoscopy 2012; 44: $136-137$

Bibliography

Endoscopy 2021; 53: E457-E458

DOI 10.1055/a-1339-5964

ISSN 0013-726X

published online 4.2.2021

(c) 2021. Thieme. All rights reserved.

Georg Thieme Verlag KG, Rüdigerstraße 14,

70469 Stuttgart, Germany 